\title{
Magnetic-Field-Induced Reorientation in Single Crystalline Ni-Mn-Ga Foil Actuators
}

\author{
F. Khelfaoui ${ }^{1}$, Srinivasa Reddy $\mathrm{Y}^{1}, \mathrm{M} . \mathrm{Kohl}^{1,2}{ }^{1}$, A. Mecklenburg ${ }^{3}$, and R. Schneider ${ }^{3}$ \\ ${ }^{1}$ University of Karlsruhe, IMT, Germany \\ ${ }^{2}$ Forschungszentrum Karlsruhe, IMT, Germany \\ ${ }^{3}$ Helmholtz-Zentrum Berlin, Germany
}

\begin{abstract}
The coupled magnetic and mechanical properties of single crystalline Ni-Mn-Ga foil actuators are investigated for decreasing thicknesses in the range from $200 \mu \mathrm{m}$ down to $60 \mu \mathrm{m}$. The foils exhibit a narrow martensitic phase transformation above room temperature below the Curie temperature of 369 K. Single crystalline Ni-Mn-Ga beam actuators are fabricated by laser micromachining. As-prepared specimens show enlarged twinning stress and thus no measurable magneto strain. Therefore, several training methods are adapted to the quasi-two-dimensional geometry of the foils. As compression training can no longer be applied in lateral direction of thin foils, more sophisticated magneto-thermo-mechanical treatments are required. We show that the low twinning stress of the bulk reference specimens can be recovered. Magnetization experiments and the direct observation of magneto strain on trained foil actuators show different degrees of reorientation up to $1 \%$ depending on the training method. The observed partial reorientation effect is discussed in terms of the foil geometry and training parameters.
\end{abstract}

\section{Introduction}

Ferromagnetic shape memory alloys (FSMAs) have attracted significant attention world-wide due to their unique magneto-mechanical properties. These materials offer large shape changes resulting either through a magnetically induced phase transformation from austenite to martensite or through magnetically induced reorientation of martensite variants connected by easily movable twin boundaries [1]. Bulk Ni-Mn-Ga single crystals for instance, reach magneto strains up to $10 \%$ at moderate magnetic field [2], which results from high magnetocrystalline anisotropy and low twinning stress in combination with a ferromagnetically ordered martensitic structure above room temperature [3].

The large magneto strains and the option of remote actuation by a magnetic field at large bandwidth make FSMA materials highly interesting for microsystems applications. Up to now, several microactuators based on polycrystalline Ni-Mn-Ga films have been developed [4-6]. Intense research currently focuses on the fabrication of epitaxial FSMA films. However, magnetically induced reorientation has been reported only in a few cases [7-10]. In order to achieve magneto-strain effects similar to bulk materials, free-standing films with improved crystallinity and microstructures are demanded.

In addition to the bottom-up approach of thin film deposition, also the alternative approach of thickness reduction of a FSMA single crystal to thin foils is currently being investigated [11,12]. The preliminary results indicate that the technologies for thickness reduction can affect the twinning stress and thus impede variant reorientation. In the following, thermo-mechanical training methods are presented to recover the low twinning stress of the starting bulk specimen after thickness reduction. Then, video microscope investigations are presented to directly observe the magneto-strain effect of the foil actuators.

This is an Open Access article distributed under the terms of the Creative Commons Attribution-Noncommercial License (http://creativecommons.org/licenses/by-nc/3.0/), which permits unrestricted use, distribution, and reproduction in any noncommercial medium, provided the original work is properly cited. 


\section{Ni-Mn-Ga foil specimens}

Starting from a $\mathrm{Ni}_{49} \mathrm{Mn}_{31} \mathrm{Ga}_{20}$ bulk single crystal, the foil specimens are prepared by electrical discharge machining followed by polishing [11]. The Ni-Mn-Ga single crystals have been grown by a SLAg Refinement and Encapsulation (SLARE) technique. Compared with the Bridgman growth method, this new technique allows producing a single crystal with low impurities and with extremely small mosaic spread. Plates of $1 \mathrm{~mm}$ thickness are initially cut from a bulk singe crystal along the (100) planes and then thinned down by electropolishing, wet-mechanical abrading and chemo-mechanical polishing to obtain the final foil thickness. Various foil thicknesses have been prepared in the range of $200 \mu \mathrm{m}$ and $60 \mu \mathrm{m}$. Subsequently, different lateral dimensions of the foils of $9 \mathrm{~mm} \times 3.25 \mathrm{~mm}$ and $5.45 \mathrm{~mm} \times 3.25 \mathrm{~mm}$ have been realized for tensile and magnetization measurements. The chemical composition of the electropolished single crystal foils are determined by EDX to be $\mathrm{Ni}_{50.60} \mathrm{Mn}_{28.33} \mathrm{Ga}_{21.06}$ (at.\%) and $\mathrm{Ni}_{50.63} \mathrm{Mn}_{28.74} \mathrm{Ga}_{21.62}$ (at.\%) for $200 \mu \mathrm{m}$ and $60 \mu \mathrm{m}$ thick foils, respectively. The X-ray diffraction pattern using $\mathrm{Cu}-\mathrm{K}_{\alpha}$ radiation of the $\mathrm{Ni}-\mathrm{Mn}-\mathrm{Ga}$ foil of $200 \mu \mathrm{m}$ thickness shows a strong and narrow (400) diffraction peak at room temperature, which is due to $5 \mathrm{M}$ tetragonal martensite [11]. This result is similar to the martensite structure of the initial bulk single crystal [13].

For the Ni-Mn-Ga foil of $60 \mu \mathrm{m}$ thickness, a small and broad peak is observed, which suggests that the crystal structure is probably associated to either $7 \mathrm{M}$ or non-modulated (NM) martensite [11] and that the preparation process affects the microstructure of the foil.

The martensitic transformation and ferromagnetic transition temperatures of the single crystalline Ni-Mn-Ga thin foils were studied by means of differential scanning calorimetry (DSC), electrical resistance and temperature-dependent low field magnetization. In DSC measurements, two narrow and sharp peaks are observed upon heating at $327 \mathrm{~K}$ and upon cooling at $335 \mathrm{~K}$, which are associated to the martensiteaustenite and the corresponding reverse transformation, respectively. In addition, kinks occur at about 370 $\mathrm{K}$ upon cooling and heating, due to the ferromagnetic transition [11].

The mechanical performance of as-received Ni-Mn-Ga foil specimens is determined by tensile tests at various temperatures. Figure 1 shows a typical stress-strain characteristic of a single-crystalline Ni$\mathrm{Mn}-\mathrm{Ga}$ foil of $200 \mu \mathrm{m}$ thickness at room temperature. An initial elastic response occurs below $3 \mathrm{MPa}$, which corresponds to a stiffness coefficient of $500 \mathrm{MPa}$. Upon further loading, a quasi-plastic behavior is observed. The twinning stress $\sigma_{\mathrm{tw}}$ is determined to be $5.5 \mathrm{MPa}$. The corresponding Ni-Mn-Ga bulk single crystal specimens exhibit a much lower twinning stress of $1.1 \mathrm{MPa}$ in compression mode, which even further decreases upon heating to about $0.3 \mathrm{MPa}$ at $323 \mathrm{~K}$ [13]. Obviously, the preparation of the foils introduces defects, which impede the mobility of twin boundaries.

Single-crystalline Ni-Mn-Ga foils of only $60 \mu \mathrm{m}$ thickness show an even higher twinning stress up to $17 \mathrm{MPa}$ [11]. In addition, a maximum strain of $10 \%$ is observed, which is usually observed for $7 \mathrm{M}$ and non-modulated martensite [15]. The high twinning stress could be due to built-in defects caused by foil preparation. On the other hand, specimens with non-modulated microstructure are also known to exhibit a relatively large twinning stress in the order of $20 \mathrm{MPa}$ [15].

Figure 2 shows a typical room temperature magnetization measurement along two in-plane directions and the out-of-plane direction of a single crystalline foil of $200 \mu \mathrm{m}$ thicknesses in as-received state. The measurements are performed by means of a superconducting quantum interference device (SQUID). No biasing stress is applied in the experiment. The curves are not corrected for demagnetization. The in-plane magnetization curve measured along the X-direction (see inset of Figure 2) shows two slopes, an initial slope at very low critical field of about 0.04 Tesla followed by a second slope with a saturation field at 0.7 Tesla. This result suggests that the sample is in a multi-variant state. Comparing the two inplane magnetization curves measured along X-and Y-directions, it is obvious that the $200 \mu \mathrm{m}$ thick foil exhibits a preferential orientation with the easy c-axis being along the $\mathrm{X}$-direction. A small hysteresis is observed in the first quadrant along the X-direction. No reorientation effect is observed due to the high twinning stress and the relatively low anisotropy energy.

\section{Thermo-mechanical training}

Bulk specimens are usually subjected to initial compressive training in order to align the martensite variants and to improve the mechanical performance. Compression of foils or thin films in lateral direction, however, 


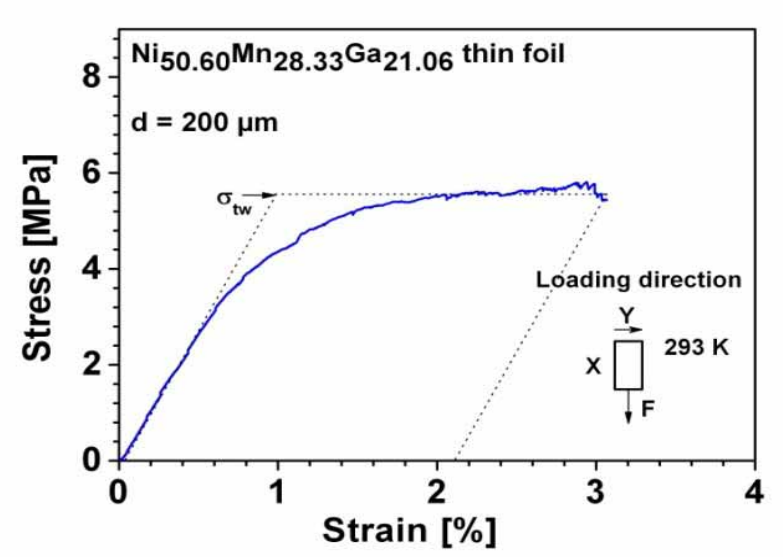

Fig. 1. Tensile stress-Strain characteristic of a singlecrystalline Ni-Mn-Ga foil of $200 \mu \mathrm{m}$ thickness in asreceived state observed at room temperature.

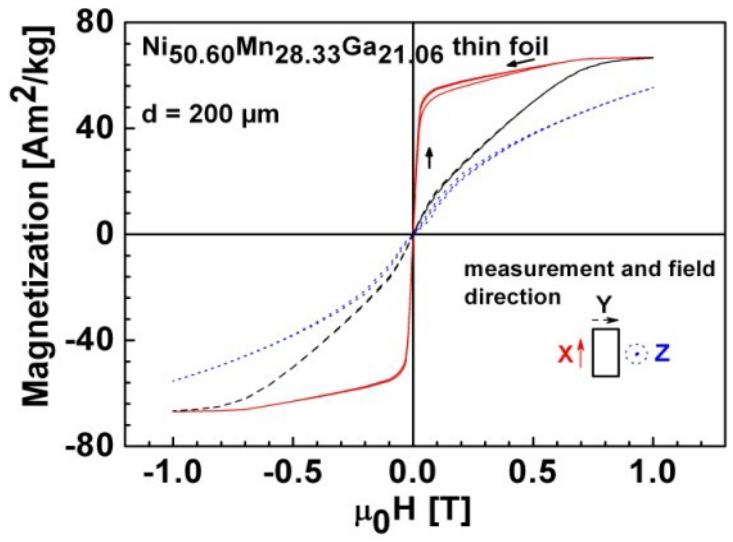

Fig. 2. Magnetization characteristics of an as-received single crystalline foil of $200 \mu \mathrm{m}$ thickness.

is not appropriate as buckling effects are likely to occur. Thus, compressive loading may only be applied perpendicular to the foil surface, which appears to be increasingly problematic for decreasing thickness as large contact forces inhibit variant reorientation. Therefore, alternative ways of training the foils have been developed taking into account their quasi-two-dimensional constraints. In the present investigation, two different thermo-mechanical training procedures have been developed as illustrated in Figure 3. The tensile load is applied along the long side of the Ni-Mn-Ga foil specimen close to the austenite finish temperature at a stress level above the stress plateau (Figure 3a). Then, the foil is subjected to a thermal cycling procedure through the phase transformation temperature regime. This training method allows the preferential alignment of the long a-axis along the tensile direction. Alternatively, also training under compression load has been investigated for different stress levels in order to determine possible limits due to contact forces (Figure 3b). In this case, pressure is applied by two copper plates with a smooth surface finish to minimize friction effects at the interfaces. The compressive load is applied close to the austenitic finish temperature to induce a quasi-plastic deformation at a stress level above the stress plateau.

(a)

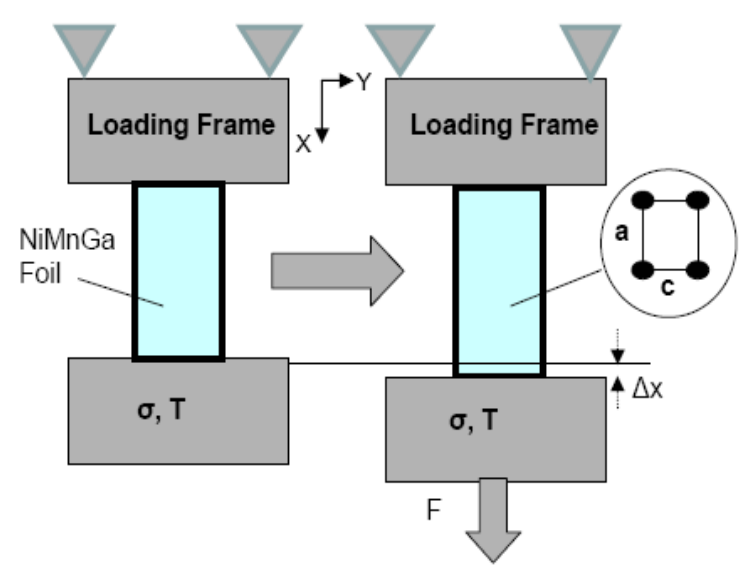

(b)

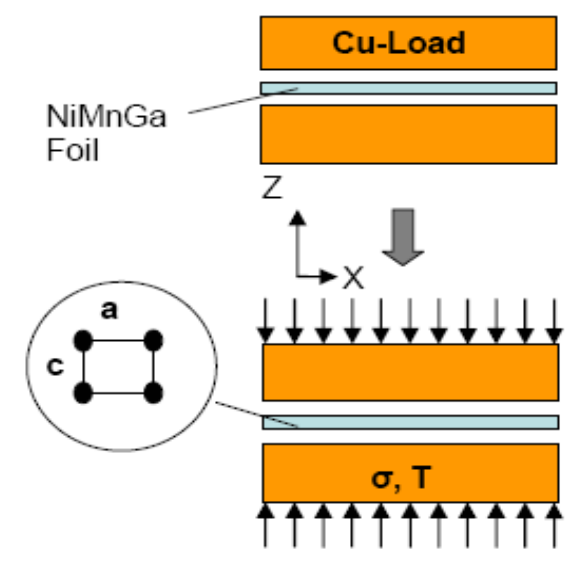

Fig. 3. Schematic of the thermo-mechanical training procedure: (a) tensile training, (b) training under compression.

By tensile training of the single crystalline foil of $200 \mu \mathrm{m}$ thickness, the twinning stress can be significantly reduced. Figure 4 shows a stress-strain characteristic measured in tensile mode along the X-direction of the foil specimen at $323 \mathrm{~K}$. Before this measurement, a magnetic field of 0.85 Tesla has been applied in Ydirection to induce a c-axis orientation of the martensite variants in Y-direction. A very low twinning stress 
of about $0.3 \mathrm{MPa}$ is observed, which is comparable to the results of the corresponding Ni-Mn-Ga bulk single crystal [13]. We therefore conclude that the tensile training method allows the complete recovery of the low twinning stress of the bulk reference.

\section{Magnetic-field-induced reorientation}

Figure 5 shows a typical in-plane magnetization characteristic of single-crystalline Ni-Mn-Ga foil of 200 $\mu \mathrm{m}$ thickness at room temperature after tensile training. The magnetic field is applied along the hard axis. The characteristic is not corrected for demagnetization. An initial linear increase of magnetization occurs due to rotation of magnetization towards the hard axis. At the critical magnetic field of about 0.35 Tesla, a jump-like behavior is observed indicating the onset of reorientation of the martensite variants. The low switching field is consistent with the low twinning stress observed in Figure 4. The jump in magnetization is accompanied by a hysteresis loop of magnetization in the first quadrant during the first cycle of magnetic field. This is because the martensite variants remain aligned upon decreasing the magnetic field at zero biasing stress. Incomplete jumps and partly developed hysteresis widths are also observed for Ni-Mn-Ga foil specimens of smaller thickness indicating a partial reorientation of the martensite variants.

In all cases, only a partial reorientation effect has been observed. One reason is that tensile training only acts on one of the two long crystallographic axes and thus only allows partial prealignment of the martensite variants. Consequently, only a fraction of martensite variants contributes to the reorientation effect. Furthermore, pinning of twin boundaries at internal defect cannot be excluded. Depending on the

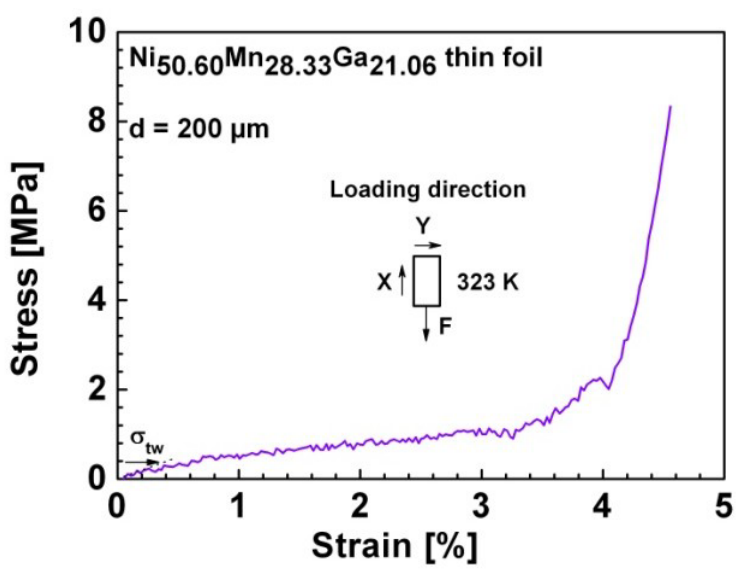

Fig. 4. Tensile stress-strain characteristic of a singlecrystalline Ni-Mn-Ga foil of $200 \mu \mathrm{m}$ thickness after tensile training observed at $323 \mathrm{~K}$.

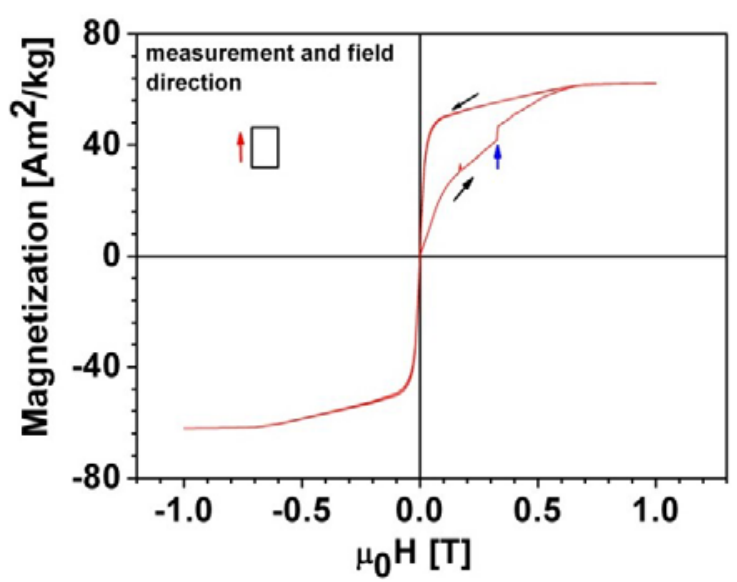

Fig. 5. In-plane magnetization characteristic of a singlecrystalline Ni-Mn-Ga foil of $200 \mu \mathrm{m}$ thickness after tensile training.

deformation energy required to overcome the pinning of the twin boundaries, reorientation may not be finished before sample saturation. Additional application of a biasing stress may help to increase the fraction of martensite variants contributing to the reorientation effect, which remains to be tested in further experiments.

Preliminary simulations of magnetization characteristics of the single crystalline Ni-Mn-Ga foils of $200 \mu \mathrm{m}$ thickness by a FSMA macromodel have been performed assuming different initial variant distributions [15]. Assuming an initial equal distribution of variants being aligned either in-plane or out-ofplane perpendicular to the tensile loading direction, we find that about $10-20 \%$ of the variants have switched into the field direction. Probably, the variants with initial out-of-plane orientation of c-axis are most likely to contribute to the switching because this orientation is energetically less favorable compared to the in-plane orientation.

After applying training under compression perpendicular to the plain of the Ni-Mn-Ga foils partial reorientation is observed to a similar extent. For compression loads above $8 \mathrm{MPa}$, however, the frictional forces dominate and, thus, inhibit reorientation. 


\section{Ni-Mn-Ga foil actuation}

The magnetic field-induced reorientation in tensile $\mathrm{Ni}-\mathrm{Mn}-\mathrm{Ga}$ foil actuators is investigated in a homogenous magnetic field by directly observing the deflection of the freely movable end of the actuator by a video microscope. The magnetic field is ramped step-wise between 0 to 0.85 Tesla, while applying the field direction either parallel or perpendicular to the long axis of the foil actuator. Figure 6 shows a sequence of photos taken during the increase and subsequent decrease of magnetic field for a single crystalline foil actuator of $200 \mu \mathrm{m}$ thickness at room temperature. A reference marker is used to eliminate relative movements of the setup with respect to the foil actuator. Below the critical magnetic field, the magneto strain keeps below the resolution of the measurement set-up of about $3 \mu \mathrm{m}$. Upon further increase of the magnetic field, the actuator suddenly contracts by about $35 \mu \mathrm{m}$ and then remains deformed until the maximum field of 0.85 Tesla is reached. No reversible effect is observed when the magnetic field is switched off.

The corresponding magneto-strain characteristic is shown in Figure 7. The contraction of $35 \mu \mathrm{m}$ corresponds to about $0.5 \%$ magnetostrain. Thus, we conclude that about $10 \%$ of the variants contribute to the magneto-strain effect, which is in qualitative agreement with the partial reorientation effect observed in magnetization experiments. After training the Ni-Mn-Ga foil actuators of $200 \mu \mathrm{m}$ thickness under compression, the fraction of contributing variants to the magneto-strain effect remains even lower then $10 \%$. Figure 7 also includes the magneto-strain characteristic of a Ni-Mn-Ga foil actuator of $60 \mu \mathrm{m}$ thickness. Due to the small actuator size only training in compressive mode has been possible. In this case, a maximum magnetostrain of $1 \%$ is observed for an optimum compressive load of about $8 \mathrm{MPa}$. Upon

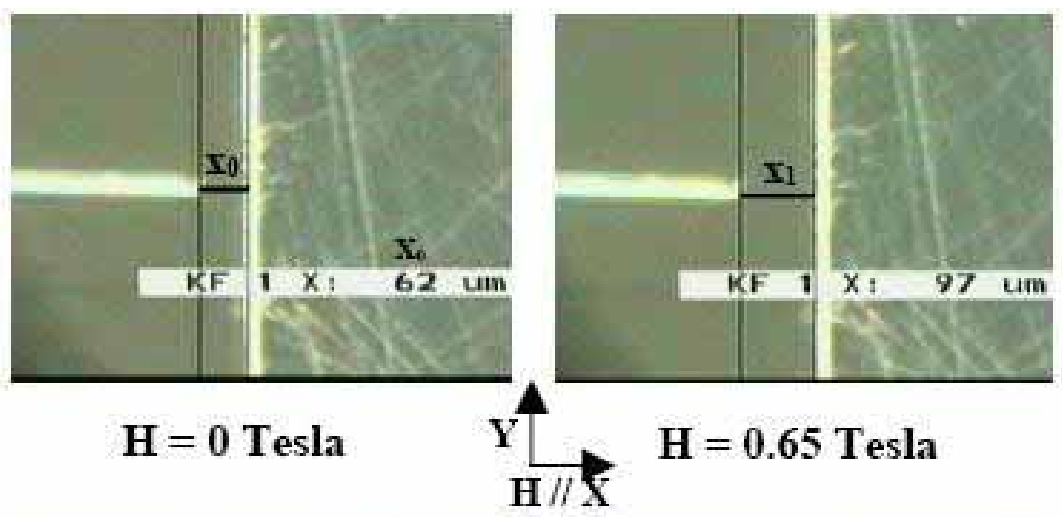

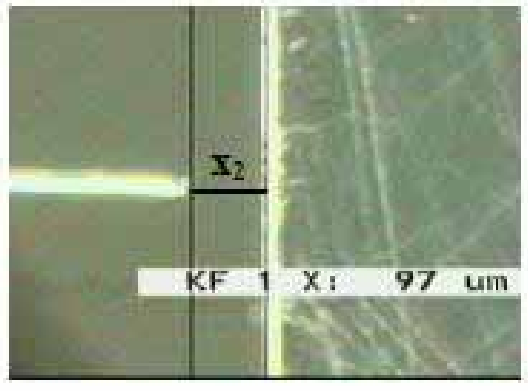

$\mathbf{H}=\mathbf{0 . 8 5}$ Tesla

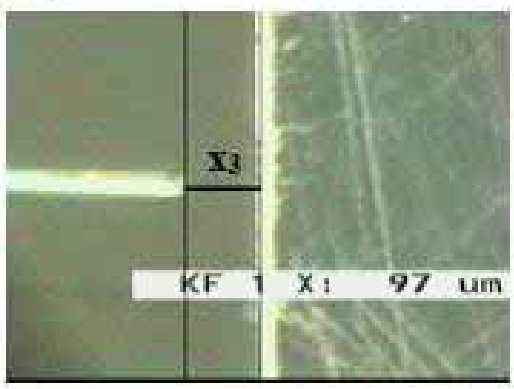

$\mathbf{H}=\mathbf{0}$ Tesla

Fig. 6. Photos of the front end of a single crystalline Ni-Mn-Ga foil actuator of $200 \mu \mathrm{m}$ thickness taken by a video microscope showing magnetic field-induced deformation. 


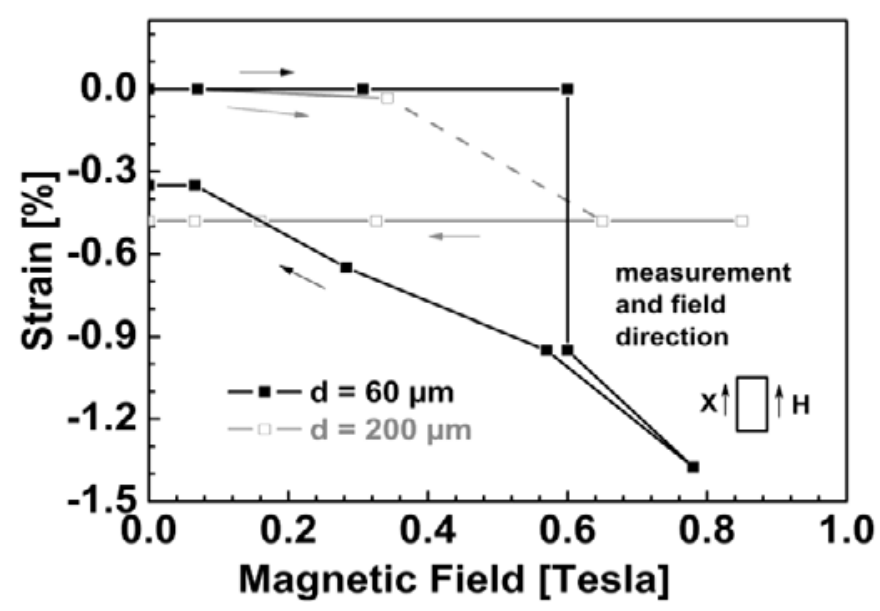

Fig. 7. Magneto-strain characteristics of single crystalline Ni-Mn-Ga foil actuators of $200 \mu \mathrm{m}$ and $60 \mu \mathrm{m}$ thickness.

decreasing the magnetic field, however, the strain is partially reset which indicates elastic deformation effects associated with reorientation.

So far, the magneto-strain experiments have been performed without bias stress. It is expected that stress assistance will help to increase the fraction of variants contributing to the switching effect.

\section{Conclusions}

The effect of magnetic-field induced reorientation has been studied in single crystalline Ni-Mn-Ga foil actuators with thicknesses ranging from $200 \mu \mathrm{m}$ and $60 \mu \mathrm{m}$, which have been prepared by thickness reduction of bulk Ni-Mn-Ga single crystals, micromachining and integration on a substrate. The Ni-Mn-Ga foil specimens show ferromagnetic martensites at room temperature. In as-received state, the Ni-Mn-Ga foils exhibit a relatively high initial twinning stress compared to the initial Ni-Mn-Ga bulk single crystals indicating an impact of thickness reduction on the microstructure of the foils. It is shown, however, that the low twinning stress of the bulk reference actuators is recovered after thermo-mechanical training, which has been adapted to the quasi-two-dimension geometry. After training, partial magnetic-field-induced reorientation up to $1 \%$ is observed by direct observation of actuator deformation at zero bias stress.

Further improvements of the methods of foil preparation as well as of the training procedures will be required to achieve a bulk-like actuation performance. In particular, future investigations will address more advanced training procedures including the combined effects of magnetic field, stress and temperature. In addition, the actuation performance will be studied in the presence of a stress bias.

\section{Acknowledgment}

The Presented work receives research funding from Deutsche Forschungsgemeinschaft (DFG) and is a part of the priority program SPP1239

\section{References}

[1] For a recent review see, e.g., A.N. Vasil'ev, V.D. Buchel'nikov, T. Takagi, V.V. Khovailo and E.I. Estrin: Physics-Uspekhi 46 (6) (2003) 559-588.

[2] A. Sozinov et al., "Giant magnetic-field-induced strain in NiMnGa seven-layered martensitic phase," Appl. Phys. Lett., 80 (2002), 1746-1748. 
[3] O. Heczko, A. Sozinov, and K. Ullakko, "Giant field-induced reversible strain in magnetic shape memory NiMnGa alloy," IEEE Transactions on Magnetics, 36 (5) 2000, 3266-3268.

[4] M. Kohl, Y. Liu, B. Krevet, S. Dürr and M. Ohtsuka, "SMA microactuators for microvalve applications", J. Phys. IV France 115 (2004) 333-342.

[5] M. Kohl, D. Brugger, M. Ohtsuka and T. Takagi, "A novel actuation mechanism on the basis of ferromagnetic SMA thin films", Sensors and Actuators 114/2-3 (2004) 445-450.

[6] M. Kohl, D. Brugger and B. Krevet, "A Ferromagnetic shape memory actuator designed for large 2D optical scanning", Sensors and Actuators A 135 (2007) 92-98.

[7] J. W. Dong, J. Q. Xie, J. Lu, C. Adelmann, C. J. Palmstrøm, J. Cui, Q. Pan, T. W. Shield, R. D. James, $\mathrm{S}$. McKernan, "Shape memory and ferromagnetic shape memory effects in single-crystal $\mathrm{Ni}_{2} \mathrm{MnGa}$ thin films", J. Appl. Phys. 95(5) (2004) 2593-2600.

[8] M. Thomas, O. Heczko, J. Buschbeck, U.K. Rößler, J. McCord, N. Scherbaum, L. Schultz and S. Fähler, "Magnetically induced reorientation of martensite variants in constrained epitaxial Ni-Mn-Ga films grown on $\mathrm{MgO}(001)$ ), New J. of Phys. 10 (2008) 23040-1-20.

[9] G. Jakob, T. Eichhorn, M. Kallmayer, and H. J. Elmers, "Correlation of electronic structure and martensitic transition in epitaxial $\mathrm{Ni}_{2} \mathrm{MnGa}$ films," Phys. Rev. B76 (2007) 174407.

[10] O. Heczko, M. Thomas, R. Niemann, L. Schultz and S. Fähler, "Magnetically induced martensite transition in freestanfding epitaxial Ni-MN-Ga films“, Appl. Phys. Lett. 94 (2009) 152513.

[11]F. Khelfaoui, M. Kohl, V. Szabo, A. Mecklenburg and R. Schneider, "Development of single crystalline Ni-Mn-Ga foil microactuator", Proc. ICOMAT 08, Santa Fe, USA, (2008).

[12] O. Heczko, A. Soroka and S.P. Hannula, "Magnetic shape memory effect in thin foils", Appl. Phys. Lett. 93, (2008) p. 022503.

[13]K. Rolfs, A. Mecklenburg, J.-M. Guldbakke, R.C. Wimpory, A. Raatz, J. Hesselbach and R. Schneider, J. Magn. Magn. Mater. (2008), doi:10.1016/j.jmmm.2008.10.023.

[14] O. Heczko, L. Straka, and K. Ullakko, "Relation between structure, magnetization process and magnetic shape memory effect of various martensites occurring in Ni-Mn-Ga alloys," J. Phys. IV France, 112, (2003), 959-962.

[15] B. Krevet, M. Kohl and S. Seelecke, "Finite element simulation of magnetic shape memory microactuators", Proc. ICOMAT 08, Santa Fe, USA, (2008). 